$$
\begin{gathered}
\text { Espacio y Desarrollo } \mathrm{N}^{\circ} 28,2016 \text {, pp. 7-23 (ISSN 1016-9148) } \\
\text { https://doi.org/10.18800/espacioydesarrollo.201601.001 }
\end{gathered}
$$

\title{
El ESTAdo ACTUAL DE LA GESTIÓN DE LA INVESTIGACiÓN AMBIENTAL en el Perú
}

\author{
Ana Sabogal Dunin Borkowski* \\ Centro de Investigación en Geografía Aplicada \\ Pontificia Universidad Católica del Perú \\ asabogal@pucp.pe
}

Fecha de envío: 27 de mayo de 2016 Fecha de aceptación: 24 de junio de 2016

\begin{abstract}
ReSUMEN
El presente artículo revisa la situación actual de la gestión de la investigación ambiental en el Perú, así como los esfuerzos nacionales para mejorar la misma. Se resaltan las características peculiares y la metodología que deben considerarse para que la investigación ambiental se incremente. Asimismo, se revisan los roles institucionales y se resaltan los avances de cada uno para plantear mejoras en la gestión. Igualmente, se destacan y discuten las implicancias del aumento del presupuesto destinado a la investigación en el Perú y a la formación de nuevos investigadores. Además se describe la gestión y las estrategias que se han llevado a cabo para mejorar los estándares de la investigación desde 2012. Por último, se presenta una propuesta para la mejora de la gestión de la investigación ambiental.
\end{abstract}

Palabras clave: investigación, ambiente, gestión, Perú, ciencias ambientales.

\section{Environmental Research Management in Peru}

\section{Abstract}

The article looks over the situation and management of environmental research in Peru. The national policy to increase and improve research in environmental science is presented and discussed. The peculiar characteristics and methodology of environmental research that need to be considered in order to increase this type of academic investigation are presented, and the institutional roles and their progress are revised. The implications of the policy to increase the budget for both research and the training of new researchers are reviewed and discussed.

\footnotetext{
PhD en Ciencias Naturales por la Universidad Técnica de Berlín, Alemania.
} 
The management plan and strategy adopted since 2012 for reaching the research standard, number of investigations and researchers set by national policy will be addressed. Finally, a proposal to improve the environmental research management is presented

Key words: Research, environment, management, Peru, science.

\section{LA INVESTIGACIÓN AMBIENTAL}

El objeto de la investigación ambiental es el ambiente, que consta de la vegetación, fauna, suelo, agua y aire; los cuales interactúan entre sí dando lugar a los ecosistemas, cuencas y clima. La investigación ambiental es parte de las ciencias de la naturaleza y por lo mismo requiere de datos primarios sobre el ambiente que deben ser recolectados a través del trabajo de campo. Estos deben ser a menudo interpretados y analizados en el laboratorio. La metodología de estudio es compleja, ya que recoge elementos de diversas metodologías de investigación y ramas de las ciencias. De esta manera, el ecólogo estudia las plantas y animales en su medio, más debe también considerar y recolectar datos sobre clima, suelo, agua, geografía y, a menudo, datos sociales y económicos. El geógrafo basa su investigación en el medio físico o territorio, integrado a la organización de la población existente en el lugar, tratando de encontrar los parámetros de su distribución. El investigador en cambio climático trabaja estrechamente con simulación de datos, estadísticas y diversos aspectos, enfocándose en la meteorología o bien en los aspectos geológicos y paleontológicos que le permitan contar con series de datos suficientes para inferir conclusiones. Los biólogos si bien realizan su trabajo en el laboratorio, requieren de muestras de campo para poder iniciar su trabajo. El meteorólogo basa su estudio en el clima, requiriendo trabajar con estaciones meteorológicas en campo, series de tiempo y estadísticas que le permitan predecir de manera adecuada el comportamiento del clima. Los impactos ambientales pueden ser estudiados desde varias aristas, como por ejemplo desde la prevención de riesgos estudiada por los geógrafos o la remediación estudiada por ecólogos, biólogos y químicos ambientales. La mitigación y adaptación a los cambios, consecuencia de los impactos ambientales, deben plantearse desde la perspectiva multidisciplinaria.

En las investigaciones ambientales a menudo se combina diferentes metodologías de investigación, empleando tanto métodos cualitativos como cuantitativos. El número de muestras y el número de repeticiones serán sustanciales para validar una investigación. Los datos que conforman una investigación ambiental a menudo combinan datos estadísticos, datos de campo sobre plantas o animales y datos poblacionales que evalúen los impactos de la población sobre los ecosistemas. De esta forma, las metodologías empleadas son tanto cuantitativas, que incluyen la toma de datos de campo, muestreo estadístico y encuesta; como metodologías cualitativas de las ciencias sociales, 
como son las entrevistas, observación participante o estudios sobre la percepción de la población. Frecuentemente se requiere recurrir a bases de datos, empleando series de tiempo y programas estadísticos con el uso de múltiples variables

Cabe resaltar que la investigación dista sustancialmente de la capacitación. Mientras la investigación debe contribuir a la ciencia, aportando un nuevo conocimiento, ya sea mediante el análisis de datos o la recolección de los mismos; la capacitación y los proyectos de extensión divulgan conocimientos. Sin embargo, a menudo se confunde ambos conceptos y se cree que basta con la recolección de datos. No obstante, para la ciencia es primordial tener en cuenta los protocolos de investigación. Sin una rigurosidad científica en la toma de datos y sin un adecuado diseño de la metodología, una investigación no se puede definir como tal. De esta manera, si en el marco de una capacitación, alumnos recolectan datos, estos no podrán servir como datos de investigación, ya que no cumplen con los protocolos de investigación y solo constituyen parte de un ejercicio. En las ciencias ambientales, como en las demás ciencias, los datos de investigación deben responder a una metodología que debe ser establecida con anterioridad a la investigación y que debe, a su turno, contar con el suficiente número de repeticiones. Para que estos datos puedan ser válidos, la metodología empleada debe ser homogénea y el número de repeticiones adecuado, de tal forma que el error de la toma de muestras sea el menor posible. Hay que distinguir, entonces, claramente entre la capacitación, la elaboración, el dictado de talleres y la investigación. Ni los datos recolectados por medio de la capacitación forman parte de la investigación, ni los capacitados la realizan. Estos pueden ser parte de un grupo de apoyo a la investigación una vez capacitados, pero no son quienes dirigen investigaciones ambientales. Los proyectos de extensión cuyo fin es mejorar la calidad de vida de la población y que ayudan al desarrollo sostenible tampoco son investigación per se, dado que estos tienen objetivos concretos, palpables y con resultados inmediatos, a diferencia de las investigaciones ambientales cuyos resultados se obtienen a largo plazo. Se requiere contar con muchas evaluaciones ambientales en diversas partes del mundo para poder llegar a una conclusión y poder elaborar las teorías que sustentan la ciencia ambiental.

Antes de realizar la evaluación de los avances realizados por la investigación ambiental en el país, se requiere distinguir con claridad lo que entendemos bajo investigación ambiental. Es necesario mencionar que la cantidad de talleres de capacitación sobre investigación no pueden ser un indicador de los avances alcanzados en el tema. Estos tampoco contribuyen a la ciencia. Los resultados deben medirse con las publicaciones científicas alcanzadas. Se requiere así evaluar fríamente los resultados de los enormes esfuerzos e inversión nacional en talleres de capacitación y distanciarlos de la investigación, no sin antes mencionar que la cantidad de esfuerzo y dinero nacional invertido en ello han sido muy grandes. Para evaluar de manera concreta se recomienda separar claramente las cuentas entre la inversión en talleres y en la investigación ambiental. 


\section{Características de la investigación AMBiental}

La investigación ambiental es compleja ya que requiere fomentar equipos de investigación y se encuentra estrechamente vinculada al trabajo de campo. Implica por lo tanto una logística compleja que asegure la toma de datos de campo. Ello implica, por un lado un presupuesto que asegure el adecuado trabajo de campo y reconozca la labor del investigador, tanto en el tiempo empleado como en el esfuerzo; y por otro, la formación de grupos de investigación sólidos que puedan dedicar el tiempo necesario a investigar. En la mayoría de casos se trata de grupos multidisciplinarios que incluyen tanto investigadores de gran experiencia (senior) como investigadores jóvenes (junior). En numerosos casos se trata de recolectar plantas, rastrear huellas de animales, tomar datos geográficos para el análisis del territorio o bien tomar muestras de espacios contaminados. Por lo tanto, se requiere no solo de la experiencia del investigador, sino también de los conocimientos locales, sin los cuales no se lograrán los objetivos. El investigador requiere también tener la habilidad para involucrar a la población local. Es justamente en esta interrelación entre los parámetros ambientales y los seres humanos que radica el éxito de la investigación ambiental. Es por ello que el investigador requiere reconocer dichos conocimientos de las poblaciones locales.

\section{Diagnóstico}

La investigación ambiental en el Perú se realiza en las universidades y en los institutos de investigación. A ello se suman algunos trabajos de extensión y recopilación de información, que aportan a la investigación. Estos son realizados por otras instituciones, principalmente organizaciones sin fines de lucro. Las empresas, si bien recopilan información —en muchos casos de primera mano— no realizan investigación. En la mayoría de casos se trata de información muy localizada que no se realiza con metodologías de investigación, ni en el número de parcelas o repeticiones apropiado para poder concluir acerca del estudio. Adicionalmente, estas se restringen a los objetivos de la empresa.

\section{Avances en la gestión de la investigación ambiental en el Perú}

Desde 2012 a la fecha se ha avanzado en el diseño y gestión de la investigación en el país, sin embargo, aún quedan muchos retos que tienen que ser superados. En este proceso lo primero que fue necesario realizar fue la actualización de un directorio nacional de investigadores, proceso que realizado por el Ministerio del Ambiente (Minam) desde 2012 a la fecha y de manera paralela por el Consejo Nacional de Ciencia y Tecnología (Concytec), durante 2013 y con metodología diferente. A la fecha se cuenta con una base de datos de los investigadores nacionales actualizada aunque aún incompleta. 
Para esta labor se requiere no solo de la voluntad de las instituciones sino también promover e incentivar la investigación. De lo contrario los investigadores no ven el interés de llenar formularios que no conllevan ningún beneficio, recargando su ya apretada agenda. Es por ello que en esta labor se requiere de la concurrencia de informáticos en coordinación con las universidades, las instituciones públicas y los investigadores. En este sentido, Concytec ha logrado ofrecer a los investigadores peruanos una base de datos de investigación actualizada, así como concursos de investigación y de publicación que resultan atractivos para los mismos. Aunque son pocos los concursos de ciencias ambientales, estos se abocan más a las ciencias puras o bien a las ciencias aplicadas de transferencia de tecnología.

Actualmente el número y calidad de las universidades es muy dispar. Una tarea pendiente es la acreditación de las universidades peruanas, labor que corresponde corroborar a la Superintendencia Nacional de Educación Universitaria (Sunedu), según la nueva ley universitaria. Ello permitirá concentrar los fondos en las universidades que cumplan con los requisitos de acreditación. Es necesario, que la acreditación de las universidades sea una acreditación internacionalmente reconocida, a fin de lograr que las universidades del Perú entren en competencia internacional y a la vez sean reconocidas en ese medio. Ello se traduciría en la formación de equipos internacionales de investigación.

En tal sentido, lo más provechoso seria que los fondos de investigación sean administrados de forma competitiva mediante una evaluación externa a las universidades. Es importante considerar que las universidades que reciben los fondos no deben evaluar los concursos de investigación nacional en los que concursen, de lo contrario serán juez y parte de los mismos. Esta deberá ser una evaluación por pares a cargo de un organismo especializado en la temática, como puede ser Concytec. Lo mismo ocurre en otros países como por ejemplo Francia o Alemania, donde el encargado del tema es un organismo del Estado

El Programa Nacional de Innovación para la Competitividad y Productividad (Innóvate Perú), juega un rol importante en la administración de los fondos de investigación, mediante la agilización en la administración de los recursos. Como se sabe, el Estado transmite el $50 \%$ del impuesto a la renta recaudado en los gobiernos regionales y locales de las zonas donde se explota el recurso minero a partir del canon. El 25\% del canon es destinado al gobierno regional donde se encuentra la mina, mientras que 5\% de este es destinado a las universidades nacionales regionales. Es necesario mencionar que los fondos del canon destinados a las universidades no se puede gastar para investigaciones ambientales por dos trabas burocráticas concretas. En primer lugar, debido a que las investigaciones deben enmarcarse dentro de los proyectos del Sistema Nacional de Inversión Pública (SNIP), los que no corresponden a una lógica de investigación por ser sumamente engorrosos, los cuales restan tiempo a los investigadores que debería 
estar destinado a la investigación. En segundo lugar, porque los fondos de investigación incluyen montos irrisorios para el trabajo de campo y viajes hacia el lugar de estudio. Cabe mencionar que según un reporte de La República (2014), basado en a su vez en un reporte de la Contraloría General de la República (2013), las universidades nacionales solo ejecutaron el $14 \%$ de recursos del canon para investigación. En el año 2014, luego de un gran esfuerzo nacional se logró subir este porcentaje al $16 \%$ (Contraloría General de la República, 2015, p. 27). Según este mismo informe de la Contraloría General de la República, para el año 2014, las universidades que recibieron mayor cantidad de dinero del canon fueron: la Universidad Nacional Intercultural de Quillabamba y la Universidad Nacional San Antonio de Abad, del Cusco, mientras que la entidad con mayor ejecución del canon fueron: el Instituto de Investigación de la Amazonía Peruana, en Loreto — que llegó a ejecutar el 71\% del presupuesto del canon—, la Universidad Nacional de San Marcos y la Universidad Nacional de Tumbes que llegaron ambas a una ejecución similar del 69\% del canon (Contraloría General de la República, 2015). Finalmente cabe cuestionar la distribución del canon para investigación orientado únicamente a las universidades regionales, política que no toma en cuenta aspectos como la trayectoria de las universidades en materia de investigación, la clasificación internacional de las mismas, el número de investigadores, el número de artículos científicos publicados, el número de investigadores con grado más alto, entre otros. Es por ello que se requiere urgentemente una profunda revisión del tema.

Es interesante pensar en priorizar focos de investigación concentrando los esfuerzos nacionales en las universidades e institutos. Sin embargo, cabe añadir que actualmente el Perú cuenta con muy pocos investigadores nacionales calificados en temas de ciencias naturales y ambiente. Por ello, y teniendo en cuenta lo mencionado, debemos preguntarnos si no debiéramos priorizar algunas universidades o institutos para que estos se conviertan en focos de investigación a nivel nacional, de tal manera que no se dispersen los esfuerzos entre muchas universidades. Indudablemente esta decisión es complicada y debe corresponder a criterios que pueden estar analizados con el directorio de investigadores ya existentes por un lado, por otro teniendo en cuenta focos de desarrollo y universidades acreditadas. En ese sentido, es interesante la Beca 18 que permite a los estudiantes con las mejores calificaciones a nivel escolar escoger la universidad en la que desean estudiar. Ello permitirá, sin duda, por un lado democratizar las universidades, permitiendo que la posibilidad de estudiar en una universidad no dependa del nivel de ingreso del alumno, sino más bien de sus capacidades y, por otro, crear competencia entre las universidades.

Una vez creados estos focos de investigación, podemos multiplicar la investigación entre otros institutos y universidades. De esta manera, los fondos podrán concentrarse y potenciarse, a fin de no dispersar esfuerzos. Entre los criterios para la priorización pueden estar la selección de los alumnos con Beca 18, el número de proyectos 
internacionales y la ubicación geográfica. Esta idea es antigua y se ha aplicado al sistema de creación de las universidades del país al crearse las universidades como la Universidad Nacional de Ingeniería (UNI) en 1876, como Escuela de Ingeniería y la Universidad Nacional Agraria, como Escuela Nacional de Agropecuaria en 1902. La creación de estas universidades correspondió a una necesidad del país y priorizó, en su época, temas necesarios para el desarrollo del país.

Es importante, teniendo en cuenta que se está iniciando el proceso de fortalecimiento de la investigación en el país, ya que los investigadores ambientales calificados son pocos y que los fondos no son infinitos, definir las prioridades de investigación a priori. Esta definición deberá ser guiada por los investigadores. En el mismo sentido, se debe resaltar que ni el Minam, ni el Concytec realizan investigación, sino que la gestionan y que el Minam debe apoyar a Concytec en esta labor pero ninguno de los dos deberán asumir el rol de investigación. Es por ello que deberán valerse de los investigadores para definir las prioridades de investigación. En tal sentido, resulta interesante la labor de Concytec en lanzar fondos de investigación para la solución de problemas concretos, sin embargo ello puede llevarnos a una gran dispersión si los criterios no han sido definidos de manera conjunta teniendo en cuenta tanto los problemas ambientales prioritarios como las capacidades de investigación existentes en el país. De igual manera, tal como indicamos, el número de investigadores ambientales es reducido, lo mismo que los temas que estos podrán afrontar.

Resumiendo, el rol del Estado es sin duda invertir y apostar por la investigación, teniendo en cuenta y comprendiendo las necesidades, los avances y los ritmos de la investigación, así como respetando la independencia, tanto de cátedra como de investigación. Resulta relevante mencionar, lo que bien se conoce, que la investigación tiene que ser independiente de las decisiones del Gobierno y que, si bien puede ayudar a la resolución de problemas ambientales nacionales, no puede servir al Gobierno para resolver problemas inmediatos. La investigación se realiza a largo plazo y requiere de una cadena de procesos que permiten el desarrollo de la investigación. Sin embargo, el Estado tiene que confiar en la investigación e invertir en ella, fomentando áreas de investigación de interés estratégico para el país. Para ello el Minam ha elaborado la Agenda de Investigación Ambiental (Minam, 2013) y el Concytec el Plan Nacional Estratégico de Ciencia, Tecnología e Innovación para la Competitividad y el Desarrollo Humano (PNCTI) 2006-2021 (Sinacyt, Concytec, 2006).

En este aspecto, el Gobierno ha venido trabajando en definir prioridades de investigación ambiental a nivel nacional, actualmente tanto Minam como Concytec cuentan con Agendas de Investigación las que han sido elaboradas conjuntamente con los investigadores nacionales. El objetivo de las agendas es concentrar los fondos en algunos temas que sean importantes a nivel nacional, en los que el Perú pueda llevar la delantera y lograr un avance importante en un mediano plazo. Para la elaboración 
de la Agenda de Investigación Ambiental, publicada a mediados de 2013, Minam ha impulsado el proceso desde 2009 (Minam, 2013). La Agenda de Investigación Ambiental es el resultado de un largo proceso de sesiones, en el que han participado investigadores de las universidades nacionales y de otros sectores. El objetivo de estas reuniones fue definir las prioridades nacionales de investigación ambiental, teniendo en cuenta los problemas ambientales más álgidos. Es en este sentido que se consideró importante, por un lado, considerar los problemas ambientales más relevantes para él país; y por otro, considerar las fortalezas que tiene el grupo de investigadores peruanos en el conocimiento del territorio y en el estudio de su biodiversidad. Fue necesario también, definir los flancos en los que no se cuenta con investigación ambiental, más sí con investigadores ambientales que pueden aportar a la solución de estos problemas.

La mirada de las universidades es muy diferente a la de los sectores y a la del Gobierno Central, dado que por un lado se trata de temporalidades diferentes, y por otro, de intereses divergentes. La discusión para la definición de las líneas de investigación contempló, un horizonte a largo plazo, en el que se pueda dar un salto cualitativo en la investigación ambiental nacional, teniendo en cuenta las limitaciones políticas que incluyen cambios constantes de prioridades y distanciándose, de esta manera, de la mirada netamente del gobierno de turno.

La Agenda de Investigación Ambiental del Minam recoge el resultado de esta discusión reuniendo las prioridades de investigación en dos ejes temáticos: conservación y aprovechamiento sostenible de los recursos naturales y de la diversidad biológica y Gestión integral de la calidad ambiental. La Agenda traza dos ejes de gobernanza transversales: compromisos y oportunidades ambientales. El horizonte de la Agenda de Investigación Ambiental va desde 2013 a 2021, tratando de esta manera de respetar los tiempos que requiere la investigación ambiental. En cada eje se han definido las líneas de investigación priorizadas. Los proyectos deberán ser elaborados por las universidades y presentados a concursos de investigación liderados por Concytec. El Concytec, en coordinación con el Minam y coincidiendo con las prioridades de la Agenda de Investigación, ha elaborado, el Plan Nacional Estratégico de Ciencia, Tecnología e Innovación para la Competitividad y el Desarrollo Humano (Sinacyt, Concytec, 2006). A continuación, en la tabla 1 se muestran los componentes de cada una de las agendas de investigación de ambas instituciones.

Otra opción igualmente válida, que puede realizarse de manera paralela a las iniciativas anteriores, es lanzar concursos de investigación competitivos, con posibilidades interesantes para los investigadores cuyos temas de investigación sean independientes a las Agendas de investigación. Es importante que la evaluación de los concursos esté a cargo de una evaluación por pares externos de investigadores extranjeros, ya que los investigadores ambientales del Perú, se conocen entre sí y no son, en este sentido imparciales. Ello, sin embargo, resulta costoso. 
Tabla 1. Ejes y componentes de las Agendas de Investigación

\begin{tabular}{|c|c|c|}
\hline Concytec & Minam & \\
\hline Ejes ambientales: & Ejes temáticos: & $\begin{array}{l}\text { Componentes de investigación: } \\
\text { a) Diversidad biológica }\end{array}$ \\
\hline 1. Agua & 1. Conservación y & b) Aprovechamiento sostenible \\
\hline Prevención de & aprovechamiento sostenible & de los recursos naturales \\
\hline desastres & de los recursos naturales y de & c) Minería y energía \\
\hline \multirow[t]{2}{*}{ Cambio climático } & la diversidad biológica & d) Bosques \\
\hline & & e) Ecosistemas marino costeros \\
\hline 2. Tecnología limpia & & $\begin{array}{l}\text { f) Recursos hídricos y suelos } \\
\text { g) Desarrollo sostenible de la }\end{array}$ \\
\hline \multirow{5}{*}{$\begin{array}{l}\text { 3. Tecnologías para } \\
\text { mitigar los impactos } \\
\text { de las actividades } \\
\text { minera, petrolera, } \\
\text { industrial y urbana }\end{array}$} & & Amazonía \\
\hline & $\begin{array}{l}\text { 2. Gestión integral de la } \\
\text { calidad ambiental }\end{array}$ & h) Cambio climático \\
\hline & & a) Calidad del agua \\
\hline & & b) Calidad del aire \\
\hline & & c) Residuos sólidos y peligrosos \\
\hline
\end{tabular}

Fuente: Minam (2013), Sinacyt, Concytec (2006). Elaboración propia.

Cabe resaltar que una de las peores cosas que puede hacer el país en investigación ambiental es destinar fondos de investigación para proyectos muy cortos y no darles continuidad. En este sentido, el reto de la investigación ambiental es grande ya que los frutos de la investigación no se logran de inmediato, por ello también una vez que nos demos cuenta de los errores habremos perdido gran cantidad de esfuerzo, dinero y tiempo. Así, por ejemplo, los resultados en la recuperación de bosques degradados recién podrán ser evaluados de manera sostenible luego de un mínimo de diez años. Las demás evaluaciones serán parciales más no definitivas. La investigación trasciende así la temporalidad de los gobiernos de turno y traza una línea de tiempo más larga.

\section{Propuesta}

Por otro lado se requiere tener claros los parámetros que definan a los investigadores ambientales nacionales, de tal manera que se puedan conformar las bases de datos teniendo en cuenta más que la voluntad, la calidad de los investigadores. Para esta labor se puede contar, por un lado, con el apoyo de las bases de datos de las universidades en red con Concytec, y por otro, del concurso de Concytec que permite contar con un registro unificado de investigadores nacionales, con títulos reconocidos y validados a nivel nacional. El Concytec ha avanzado considerablemente en esta meta, en 2013 y cuenta actualmente con la base de datos de los investigadores nacionales. 
El Minam, por su parte, también entre 2012 y 2013 ha realizado una línea base de investigadores ambientales a nivel del país. Esta línea base ha recopilado los investigadores ambientales como aquellos que realizan extensión ambiental, no teniendo una clara la distinción entre estos e incluyendo centros tecnológicos. Es necesario continuar el proceso definiendo los parámetros claros para la definición de los investigadores ambientales y sobre todo trabajar en red optimizando de esta manera los esfuerzos tanto de tiempo como de dinero.

Para la clasificación de las investigaciones e investigadores en el Perú se utiliza los códigos de la Organización de las Naciones Unidas para la Educación, la Ciencia y la Cultura (Unesco), que no consideran a las Ciencias Ambientales como tal sino a las Ciencias de la Tierra, que dejan de lado la interacción con el hombre. Sin embargo es la metodología que ha venido empleando la mayoría de instituciones, o colocando la base de datos de los investigadores nacionales en una base de datos utilizando los clasificadores de la Organización para la Cooperación y el Desarrollo Económico (OECD, por sus siglas en inglés), la que cuenta con códigos reconocidos internacionalmente y con una clasificación actualizada de las ramas de las diversas ciencias ambientales.

Se requiere además uniformizar los criterios de titulación y revalidación de títulos a nivel nacional, para ello se ha creado un nuevo organismo estatal la Superintendencia Nacional de Educación Universitaria (Sunedu). Con ello se pretende mejorar la gestión universitaria y asegurar un nivel mínimo de educación superior, además de mejorar la gestión de la inversión nacional en educación superior. En la mayoría de países, como Francia o Alemania, esta función está a cargo del Ministerio de Educación que es quien valida los títulos universitarios a nombre de la Nación.

Para potenciar la investigación en el país se requiere fortalecer la estructura de investigación en el país. Para ello se puede considerar tres modelos, no sin antes tener en cuenta que en el país se cuenta con muy pocos investigadores ambientales que posean doctorados en universidades extranjeras reconocidas a nivel mundial. Las opciones son las siguientes: o bien se apuesta por un modelo que repotencie la labor de investigación que realizan los investigadores peruanos, creando mayor competencia entre ellos mediante concursos de investigación abiertos y competitivos; o bien se continua priorizando a las universidades que poseen canon, pero que no poseen necesariamente grupos de investigadores competitivos, mediante concursos en los que pueden también participar otras universidades, formando grupos mixtos de investigación interuniversitarios. Teniendo además en cuenta que según la revista británica The Times Higher Education, ninguna de las universidades peruanas está en el ranking de las 100 mejores universidades de mundo.

Otra labor importante que si bien se encuentra avanzada, requiere mayor esfuerzo, es la creación de un Repositorio de Investigaciones Ambientales Nacionales. Este deberá 
contener las investigaciones que se realizan a nivel nacional, tanto de los investigadores peruanos como extranjeros, que han trabajado temas ambientales sobre el Perú. Se trata de un sistema que deberá estar, por un lado interconectado con las principales bibliotecas ambientales del país y por otro, también deberá actualizarse de manera automática. Actualmente la información, resultado de las investigaciones ambientales realizadas en el Perú, se encuentra dispersa. Los sistemas de los repositorios bibliográficos no se encuentran unificados, por lo que es muy difícil que pueda existir una interconexión entre las pocas bibliotecas ambientales del país. El costo de unificar los sistemas resulta elevado, lo que dificulta esta labor, siendo sin embargo una prioridad y resultando un costo muy bajo, si se tiene en cuenta el efecto multiplicador de esta labor. Una traba que requerirá de gran inversión es la baja calidad de la banda de internet. Ello se aplica también al acceso a la información y a las revistas indexadas en las que se encuentran las investigaciones recientes son sumamente caras y la mayoría de investigadores no accede a ellas. Por ello sería de gran ayuda que el Concytec incentive la mejora de la información y permita el acceso de sus investigadores a las revistas indexadas.

El Perú tiene que integrarse a la comunidad científica internacional, de lo contrario las investigaciones nacionales no tendrán la relevancia ni la validez que permitan al país no solo validar las investigaciones, sino dialogar con la comunidad científica mundial y desarrollar teorías de la investigación, de lo contrario siempre los avances serán locales. Es necesario para el país corroborar los resultados de las investigaciones ambientales a nivel mundial y comparar espacios geográficos semejantes, lo cual en su conjunto será esencial para poder construir teorías en las ciencias ambientales. Uno de los problemas del Perú es la falta comunicación de las investigaciones realizadas, en muchos casos las investigaciones se difunden solo a nivel local.

$\mathrm{Al}$ igual que en muchos otros países en desarrollo, son muy pocas las revistas científicas que se editan en el Perú y escasas están indexadas y poseen un sistema de evaluación externo a la institución. En la mayoría de casos son los miembros de la institución los que realizan la evaluación por pares. Muchas revistas peruanas no realizan evaluación por pares, además a menudo esta no es anónima. Cabe resaltar que en 2011, en el Perú solo se publicaron 84 artículos sobre temas ambientales en revistas ambientales, sin embargo esta cifra es bastante mayor a 1996 en que el número de artículos fue de 10 (Rivera, 2013). Como resultado de ello, la calidad de las publicaciones es variada, en muchos casos deficiente. Gran parte de la investigación queda en pequeñas revistas locales, y en muchos casos la metodología de investigación no se encuentra validada (Deil, Álvarez, Bauer y Ramírez, 2011). Al respecto existen muchos problemas que dificultan el acceso de comunidad científica mundial a los datos recolectados. Entre las principales dificultades, que mencionan Deil et al. (2011), que aunque mencionan los problemas específicamente para investigaciones de florística vegetal, bien pueden 
ser considerados de manera amplia para las investigaciones ambientales en general, se encuentran las siguientes:

- La nomenclatura científica de las especies no es utilizada de manera unificada, de esta manera la nomenclatura florística nacional cambia con el país y la fecha de publicación;

- La distribución de las publicaciones es limitada al ámbito nacional, en muchos casos a la región,

- Las muestras recolectadas no son homogéneas, por lo que no sirven para poder realizar comparaciones con otras recolecciones y llegar a conclusiones validas;

- Se describe la vegetación teniendo en cuenta los registros nacionales de endemismo y no los internacionales.

En consecuencia, no se cuenta con una nomenclatura unificada para los diversos campos de las ciencias ambientales. Por ejemplo, no se se dispone de nomenclaturas uniformes ni para la florística, ni para la clasificación ecológica, ni para la clasificación de suelos, ni para las coordenadas geográficas. Todo ello conlleva a que no se pueda trabajar de manera unificada y se requiera de un esfuerzo adicional para lograr bases de datos nacionales de mayor escala como puede ser un mapa unificado; además del costo que esto representaría. Es por ello que se requiere que los investigadores y el Gobierno se pongan de acuerdo en la nomenclatura a utilizarse utilizando nomenclaturas internacionales. Todo lo antes mencionado conlleva a no poder avanzar con investigaciones científicas complejas, como pueden ser las investigaciones en cambio climático global, biogeografía, evolución de las especies, la paleontología e incluso en desarrollo sostenible. De esta manera, quedan las investigaciones a nivel local. Sin embargo, todos estos temas no pueden ser vistos solo de manera nacional, reduciendo los avances de la investigación al ámbito local; se trata de que las investigaciones necesariamente tengan que considerar los avances y comparaciones mundiales.

Para lograr un repositorio ambiental que incluya la mayor cantidad de publicaciones nacionales, el Minam ha realizado dos actividades concretas: por un lado la recopilación de la información sobre investigadores ambientales nacionales existentes en el país; por otro, la recopilación de los trabajos escritos dispersos en el país. Se ha trabajado en tal sentido, realizando en el ańo 2012 y 2013, dos Encuentros de Investigadores Ambientales nacionales, y otro en 2014. Lamentablemente estos encuentros han sido descontinuados. El objetivo de los Encuentros de Investigadores Ambientales Nacionales era acercar a los investigadores nacionales entre si dando a conocer las investigaciones que cada uno de ellos realiza y la formación de un puente que permita, por un lado, visualizar las investigaciones nacionales; y por otro, fomentar equipos de investigación que incluyan a las universidades entre sí, las empresas, 
cuyo rol debe ser realzado y potenciado en cuanto a la inversión en investigación ambiental, y el Estado, cuyo apoyo es clave para el desarrollo de la investigación en el Perú. El Concytec ha avanzado considerablemente en este sentido, aumentando el número de investigadores inscritos en Concytec desde 2012, de un número de 2015 a 13000 (Reyes, 2014).

Cabe resaltar que gran parte de los trabajos de investigación ambiental en el Perú se encuentran dispersos a nivel nacional, en diversas regiones del país. En tal sentido, el Minam ha avanzado con la creación de un Repositorio Ambiental Nacional que se encuentra en sus instalaciones. Este aún requiere aproximadamente, un 50\% de trabajo de catalogación y elaboración de fichas de contenido. Es sin embargo un importante avance y quizá el más completo a nivel nacional, ya que ha incluido la información de las investigaciones realizadas incluyendo a las regiones.

Es necesario aumentar el número de investigadores calificados. Para ello un elemento clave es, por un lado que existan becas de investigación para la formación de nuevos investigadores, ello puede lograrse con becas de investigación que incluyan tanto la exoneración del pago como la manutención. Actualmente en Concytec concentra la información sobre las becas a nivel de posgrado y doctorado. Algunas universidades como la Pontificia Universidad Católica del Perú (PUCP), administra mediante convenios becas para posgrados y doctorados como son algunas becas de la cooperación alemana o bien francesa. Las becas para estudios en el extranjero deben necesariamente tener como condición que los investigadores tengan que realizar las estadías de investigación fuera de su universidad de origen permitiéndoles enfrentarse a la investigación mundial. Resulta importante mencionar que actualmente este es un elemento clave para que el nivel de los posgrados en el país pueda mejorar. Actualmente el alumno de posgrado en el Perú, además de estudiar trabaja todo el día llegando a estudiar a partir de las 6 de la tarde o bien, peor aún los fines de semana. Los resultados no pueden compararse de ninguna manera con los posgrados internacionales a tiempo completo. En este sentido el Concytec ha lanzado una convocatoria a becas completas para los posgrados en el país.

También será necesario invertir en repositorios ambientales nacionales que incluyan disponibilidad de información internacional con suscripción a las mejores revistas de investigación mundial de tal manera que tanto el investigador como el alumno tengan acceso a los resultados de investigación mundial. Para ello el país deberá necesariamente invertir. Por otro lado, se requiere la internacionalización de la investigación ambiental formando grupos de investigación internacionales, para lo cual el país deberá realizar esfuerzos que acerquen a los investigadores ambientales nacionales con los internacionales.. En este aspecto no se ha avanzado casi nada. Una posibilidad que permitiría avanzar hacia esta meta, teniendo en cuenta los criterios para las calificaciones internacionales, es aumentar el número de docentes doctores, 
con grados obtenidos en universidades externas, en las distintas universidades del Perú. Ello se puede lograr mediante el otorgamiento de becas de investigación, pero también regulando la financiación a grupos de investigación que incluyan doctores con grados obtenidos internacionalmente. Los grupos de investigación deberían incluir, en lo posible, varias universidades nacionales e internacionales, siempre que los investigadores posean publicaciones indexadas. En este sentido una medida a tomar, aunque algo drástica, podría ser financiar solo grupos de investigación universitarios que posean un porcentaje de doctores calificados internacionalmente, pudiendo conformarse de esta manera grupos de investigación múltiple o bien de universidades que trabajen con otras universidades que posean estas calificaciones.

En este aspecto es interesante mencionar tres ejemplos: el Instituto de la Amazonía Peruana (IIAP,) el Instituto Geofísico del Perú (IGP,) el Servicio Nacional de Meteorología e Hidrología del Perú (Senahmi), los tres adscritos al Minam, y el Centro Internacional de la Papa (CIP). Cada una de estas instituciones ha desarrollado considerable investigación ambiental. El IIAP ha apostado por el desarrollo sostenible de la Amazonía y aunque no cuenta con gran número de doctores, se ha asociado a instituciones internacionales como el Instituto de Investigación y Desarrollo de Francia (Institut de Recherche pour le Développement, IRD), además de otras fuentes internacionales para las investigaciones ambientales. De esta manera ha logrado interesantes propuestas de desarrollo sostenible aplicadas a la Amazonía e incluso al mercado peruano en general, como es el desarrollo de la piscicultura fluvial. El IGP ha apostado por un equipo de investigadores conformado en algo más del $50 \%$ por profesionales que poseen doctorado y exigiendo a los mismos mantener publicaciones internacionales. Para el logro de sus investigaciones trabaja, al igual que el IIAP, en asociación con instituciones internacionales a parte de contar con fondos nacionales. El CIP ha apostado por el trabajo internacional, abriendo sus investigaciones a otros tubérculos andinos y a la conservación in situ de recursos genéticos. A diferencia de estos tres, al Senahmi le falta aún dar el salto cualitativo aumentando el número de profesionales con doctorado, que permita contar con investigaciones y publicaciones internacionales reconocidas.

Finalmente, se considera que no se trata solo de contar con investigadores calificados, sino que estos deberían además tener la posibilidad de trabajar en investigación. Muchos doctores altamente calificados terminan trabajando en cooperación internacional o bien en otras labores de gestión, perdiéndose un potencial de personas altamente calificadas debido a que los sueldos de las universidades nacionales son exiguos. Asimismo, se considera que no solo se trata de la remuneración, se requiere además de una infraestructura adecuada para investigar. Ello implicaría, campos de investigación experimental, invernaderos y laboratorios debidamente equipados 
en los que puedan regularse los factores ambientales, así como equipos e insumos para la recolección adecuada durante el trabajo de campo. Se requiere aumentar el número de herbarios reconocidos que cumplan con los requisitos internacionales, los centros de investigación que posean infraestructura y laboratorios bien equipados, con equipos microscópicos electrónicos para el estudio de minerales y la decodificación genética, por ejemplo. Este aspecto ha sido contemplado por Concytec, quien ha lanzado recientemente un concurso para lograr la mejora de los laboratorios nacionales. Ello sin duda es un paso muy relevante que solo funcionará si va unido al desarrollo de la capacidad humana adecuada. En tal sentido se requiere aún avanzar con la estrategia de gestión de la investigación. Cabe resaltar que hasta ahora la mayor inversión en laboratorios se ha dado por parte de entidades privadas como la Universidad Privada Cayetano Heredia y la Pontificia Universidad Católica del Perú (esta última no cuenta con una facultad de ciencias ambientales) y principalmente, por parte de fondos internacionales que han permitido financiar los laboratorios de la Universidad Nacional Agraria La Molina o centros de investigación como la Asociación para la Conservación de la Cuenca Amazónica (ACCA) en la selva de Madre de Dios, el IIAP en la selva de Loreto, el Instituto Geofísico del Perú (IGP). Estos dos últimos, si bien reciben financiación nacional, cuentan también con fondos internacionales para investigación. La dispersión de fondos del Concytec para crear centros de excelencia, diluye la responsabilidad nacional de asumir un rol más fuerte en este tema.

Finalmente cabe mencionar que dado que el objeto de investigación ambiental nacional está ligado al vínculo entre la naturaleza y los procesos socioambientales que ello inplica, se requiere tener en cuenta las leyes respecto a la recolección de plantas, los derechos de autor de la información ambiental y el compromiso ético del investigador con la población local. Actualmente, se requiere la aceptación de la población local para poder difundir los conocimientos locales, trasmitidos de generación en generación de manera oral, reconociendo la autoría de los mismos. En tal sentido es interesante resaltar que el Instituto Nacional en Defensa de la Competencia de la Protección de la Propiedad Intelectual (Indecopi) reconoció hasta octubre de 2011, 2120 registros de los pueblos indígenas asociados a la biodiversidad basado en la ley 27811. Así, en octubre de 2013 los derechos de las técnicas empleadas por la comunidad indígena Tsachopen sobre el uso de seis plantas amazónicas: tornillo, árbol estrella, palo tintura, matico y payón, utilizadas por el pueblo yanesha, fueron reconocidos por el Indecopi. De esta manera, deberá solicitarse el permiso y remunerar a la comunidad si se desea utilizar los conocimientos indígenas sobre estas plantas. 


\section{REFERENCIAS BIBLIOGRÁFICAS}

Congreso de la República (31 de mayo de 2013). Proyecto de ley que propone una nueva ley universitaria. Recuperado el 6 de enero de 2014, de Expediente Virtual Parlamentario: http://www2.congreso.gob.pe/Sicr/TraDocEstProc/Expvirt_2011.nsf/didpley/2FAE 8CD1E206159D052579030054F2C5

Contraloría General de la República (20 de diciembre de 2013). Universidades públicas sólo ejecutaron el $14 \%$ de los recursos del canon que deben destinar a investigación cientifica. Recuperado el 3 de enero de 2014, de NOTA DE PRENSA N 133-2013CG/COM: http://www.contraloria.gob.pe/wps/wcm/connect/b272804c-97e24795-9aab-e5d19cfccd19/NP_133_2013_CG+20-12-2013.pdf? $\mathrm{MOD}=$ AJPERES\&CONVERT_TO=url\&CACHEID=b272804c-97e2-4795-9aabe5d19cfccd19

Contraloría General de la República (diciembre de 2015). Transferencia y ejecución de los recursos del Canon, Sobrecanon, Regalía Minera y Fondo de Desarrollo Socioeconómico del Proyecto Camisea (FOCAM). Periodo 2009 - julio 2015. Obtenido de REPORTE N ${ }^{\circ}$ 12-2015-CG/EST: http://doc.contraloria.gob.pe/estudios-especiales/reportes/2015/ Reporte_N012-2015-CG-EST.pdf

Deil, U., Álvarez, M., Bauer, E.-M. y Ramírez, C. (2011). The vegetation of seasonal wetlands in extra-tropical and orotropical South America. Phytocoenologia, 41(1), 1-34. http://dx.doi.org/10.1127/0340-269x/2011/0041-0491

Diario Oficial El Peruano (9 de julio de 2014). Ley No 30220. Ley Universitaria. Recuperado el 27 de 05 de 2016, de Diario Oficial El Peruano. Normas Legales. Año XXXI- N¹2914: http://www.sunedu.gob.pe/files/normatividad/LEY_UNIVERSITARIA.PDF

El Comercio (2013 de diciembre de 2013). Acreditación de universidades mejorará nivel educativo, dijo rector de la UPC. Recuperado el 06 de enero de 2014, de http://elcomercio.pe/mundo/actualidad/acreditacion-universidades-mejorara-nivel-educativodijo-rector-upc-noticia-1670768

La República (28 de agosto de 2011). Indecopi promoverá registro de conocimientos colectivos depueblos indígenas. Recuperado el 3 de Enero de 2013, de http://larepublica.pe/28-082011/indecopi-promovera-registro-de-conocimientos-colectivos-de-pueblos-indigenas

La República (1 de enero de 2014). Universidades públicas solo ejecutaron el 14\% de recursos del canon para investigación. Recuperado el 3 de enero de 2014, de http://larepublica. pe/01-01-2014/universidades-publicas-solo-ejecutaron-el-14-de-recursos-del-canonpara-investigacion

Minam (2013). Agenda de Investigación Ambiental, 2013-2021. Lima: Ministerio del Ambiente. 
Ministerio de Economía y Finanzas (9 de julio de 2001). Ley N²7506. Ley del Canon. Obtenido de https://www.google.com.pe/url?sa=t\&rct=j\&q=\&esrc=s\&source=web\& $\mathrm{cd}=1 \& \mathrm{cad}=$ rja\&uact $=8 \& \mathrm{zed}=0 \mathrm{ahUKEwjj5u}$ _ppqbOAhVL4iYKHaR_BcAQFggcM AA\&url=https\%3A\%2F\%2Fwww.mef.gob.pe\%2Findex.php\%3Foption\%3Dcom_ docman\%26task\%3Ddoc_download\%26gid\%3D6737\%26Itemid\%3D101598\%2 6lang $\% 3$

Reyes, R. M. (02 de enero de 2014). Diario Gestión. Recuperado el 03 de enero de 2014, de Concytec: «Requerimos S/. 4,000 mlls. para formación de 17,000 científicos para el bicentenario»: http://gestion.pe/tecnologia/concytec-cientificos-ciencia-peruanarequerimos-s-4000-mlls-formacion-17000-cientificos-2021-bicentenario-2085165

Rivera, N. (03 de julio de 2013). El Perú es uno de los paises de Sudamérica con menos investigación ambiental. Entrevista a Ana Sabogal. Recuperado el 02 de enero de 2014, de CEPES Rural - Artículo publicado en el Observatorio de Cambio Climático: https:// cepesrural.lamula.pe/2013/07/03/el-peru-es-uno-de-los-paises-de-sudamerica-conmenos-investigacion-ambiental/cepesrural/

Sinacyt, Concytec (abril de 2006). Plan Nacional Estratégico de Ciencia Tecnología e Innovación para la Competitividad y el Desarrollo Humano. Lima: Concytec. Obtenido de https://portal.concytec.gob.pe/images/stories/images2012/portal/areas-institucion/ pyp/plan_nac_ctei/plan_nac_ctei_2006_2021.pdf

Ukhamawa Noticias (11 de diciembre de 2013). Perú reconoce propiedad intelectual de conocimientos indígenas. Recuperado el 3 de enero de 2014, de Ukhamawa: Noticias Indígenas: https://ukhamawa.wordpress.com/2013/12/11/peru-reconoce-propiedadintelectual-de-conocimientos-indigenas/ 